\section{MATERNAL SHIFT WORK DURING PREGNANCY AND INFANT NEURODEVELOPMENT PARAMETERS AT EIGHTEEN MONTHS: RESULTS FROM TAIWAN BIRTH COHORT STUDY}

${ }^{1,2}$ Chihfu Wei* ${ }^{1,2}$ Pau-Chung Chen. ${ }^{1}$ Institute of Occupational Medicine and Industria Hygiene, National Taiwan University, Taipei, Taiwan; ${ }^{2}$ Department of Environmental and Occupational Medicine, National Taiwan University Hospital, Taipei, Taiwan

\subsection{6/oemed-2018-ICOHabstracts.409}

Introduction Maternal shift work were found to be associated with altered sex hormone, pre-term delivery and small for gestational age. Previous studies also showed shorter breastfeeding duration among mothers with shift work. However, whether these adverse effects affect future infant neurodevelopment remained uncertain. Therefore, we aimed to find the association between maternal shift work status and children's neurodevelopment parameters.

Methods Taiwan Birth Cohort Study (TBCS) was the first nationwide birth cohort study in Taiwan that follows representatively sampled mother-infant pairs in 2005. The development and exposure condition of the participants were assessed by home interview with structured questionnaires upon six and eighteen months old. Propensity score matching was used to generate subpopulation with comparable characteristics. Multivariate logistic and Cox proportional hazard regression model were applied to examine the relative risk (RR) and hazard ratio (HR) between maternal shift work status and caregiverreported neurodevelopment milestones.

Results In this study, 1751 term singletons were included in the propensity score matched cohort. Maternal shift work was associated with delay in care-giver reported gross motor neurodevelopment milestone, such as able to draw arbitrarily $(\mathrm{RR}=1.39,95 \%$ confidence interval $\mathrm{CI}: 1.05$ to 1.84 ; hazard ratio $(\mathrm{HR})=1.13,95 \%$ confidence interval $\mathrm{CI}$ : 1.02 to 1.25$)$, able to drink with both hands $(\mathrm{HR}=1.11,95 \%$ confidence interval CI: 1.00 to 1.23 ), and lower score in gross motor domain of TBCS development instrument ( $R R=1.41,95 \%$ confidence interval $\mathrm{CI}$ : 1.01 to 1.96$)$. However, the effects were attenuated after multivariate adjustment.

Conclusion This study showed possible associations between maternal shift work and delay in neurodevelopment milestone achievement at eighteen months. Although the associations were attenuated when adjusted for other parameters, further studies may be indicated to ascertain the association and the possible dose-response effect.

\section{USING THE NATIONAL HEALTH INTERVIEW SURVEYTO STUDY WORKPLACE PSYCHOSOCIAL EXPOSURES IN THE U.S}

Sara Luckhaupt. National Institute for Occupational Safety and Health, Cincinnati, OH, USA

\subsection{6/oemed-2018-ICOHabstracts.410}

Introduction The National Health Interview Survey (NHIS) is an annual, in-person health survey that is the primary source of information on the health of Americans. In 2010 and 2015, the National Institute for Occupational Safety and Health (NIOSH) sponsored sets of work-related questions in the NHIS, called Occupational Health Supplements (OHSs) in order to estimate the prevalence of workplace exposures, including psychosocial exposures, and common work-related health conditions.
Methods The 2015 NHIS-OHS included questions about several psychosocial exposures: work-life interference, worry about losing one's job, hostile work environment, job demands, job control, and supervisory support. The data are publicly available, and $\mathrm{NIOSH}$ has estimated the prevalence of all of these exposures using statistical procedures that take the complex sample design into account and applying NHIS sample adult record weights to produce nationally representative results.

Result The 2015 NHIS-OHS collected data on more than 19000 employed adults, representing almost 158 million American workers. The overall prevalence of work-life interference was $25.5 \%$, ranging from $23.9 \%$ among workers employed in wholesale and retail trade to $31.1 \%$ among workers employed in transportation, warehousing and utilities industries. The overall prevalence of worry about losing one's job was $11.0 \%$, ranging from $10.0 \%$ among those employed in services industries to $29.0 \%$ among those employed in mining. The overall prevalence of a hostile work environment was $6.9 \%$, ranging from $1.5 \%$ among those employed in agriculture, forestry, and fishing to $9.9 \%$ among those employed in healthcare and social assistance. High job demands were reported by $14.4 \%$, low job control was reported by $13.7 \%$, and low supervisory support was reported by $9.9 \%$ of workers.

Discussion Many U.S. workers experience workplace psychosocial exposures that may adversely impact their health, and the prevalence of these exposures varies by industry. Workplace or industry-specific interventions, developed with worker participation, may be warranted.

\section{RAISING THE BAR: WATER, SANITATION \& HYGIENE AT WORKPLACE}

Rishikesh Naik*. Aditya Birla Group, Mumbai, India

\subsection{6/oemed-2018-ICOHabstracts.411}

Introduction UN's Sustainable Development Goals (SDG) 6.1 and 6.2 aim to achieve universal access to water, sanitation and hygiene (WASH) by 2030. Today, as estimated, over 1.8 billion people are still without access to safe drinking water and 4.1 billion lack access to adequate sanitation. There is a compelling and clear economic case for businesses to demonstrate leadership by addressing this situation. Many businesses have operations, employees, contractors and customers in countries lacking access to WASH provisions. Economic, social and environmental impacts can cause illness or fatalities, impair productivity, and restrict markets for some products and services. Aditya Birla group (ABG) - a 41 billion USD multinational conglomerate, recognise this and is committed to play its role by signing World Business Council for Sustainable Development (WBCSD) WASH Pledge.

Methods WASH Self-Assessment Questionnaire (SAQs) from WBCSD were framed in specific sustainability software and launched in 2015 to understand the current level of WASH provisions available at premises under direct company control against the international standards. Awareness on required aspects of WASH provisions were provided based on gaps identified and best practices within the groups were shared. Periodic update on status were made available to decision makers along with Key focus areas. The scores were reviewed in business review meeting. 